\title{
An investigation into whether participation in a four week dietary intervention can modify reported barriers towards fruit and vegetable consumption
}

\author{
C. Rooney ${ }^{1}$, M. C. McKinley¹, K. M. Appleton ${ }^{2}$, I. S. Young ${ }^{1}$, A.J. McGrath ${ }^{1}$, C. R. Draffin ${ }^{1}$, \\ S. Bhattacharya ${ }^{1}$, L. L. Hamill, S. E. C. M. Gilchrist ${ }^{1}$, C. E. Neville ${ }^{1}$ and J. V. Woodside ${ }^{1}$ \\ ${ }^{1}$ Centre for Public Health, Queen's University Belfast, Belfast, BT12 6BJ, UK and ${ }^{2}$ Department of Psychology, \\ Bournemouth University, Poole, Dorset, BH12 5BB, UK
}

Despite recommendations to consume five portions ( $400 \mathrm{~g}$ ) of fruit and vegetables (FV) per day, current intakes remain suboptimal ${ }^{(1)}$. Whilst various studies have identified factors that consumers anticipate would be important barriers towards eating $\mathrm{FV}^{(2,3)}$, little research has examined the barriers that are experienced when dietary change is actually implemented. Furthermore, the potential ability for FV interventions to modify such barriers is currently under researched. Hence this was the main aim of the current study.

Thirty low FV consumers, aged 20-65 years, were recruited to take part in a randomised controlled feeding study. Participants were randomised to one of three diets ( $\leq 2$ portions FV/day; 5 portions FV/day; 8 portions FV/day) for four weeks. A questionnaire, which has been used previously in a study examining barriers towards FV, was distributed pre and post intervention. It comprised 20 closed-response questions and three open-response questions, and assessed five factors (liking, willingness, ease, difficulties and awareness) which represent barriers towards FV consumption. Between and within-group changes in barriers were assessed using the Kruskal-Wallis Test and Wilcoxon Signed Rank Test respectively.

As illustrated in the table below, no significant changes in barriers were detected between the diet groups from pre to post intervention within the closed-response questions (all $\mathrm{P}>0 \cdot 05$ ). However, liking for FV was significantly greater within the 8 portions FV/day group at week 4 compared to week $0(\mathrm{P}=0.007)$. Responses from the open-ended questionnaire items varied slightly from pre to post intervention. For example, some of the barriers mentioned at baseline were not mentioned at week 4 , including that FV were 'inconvenient to eat on-the-go'. Similarly, new barriers were mentioned post intervention, including 'knowledge on how to incorporate FV into meals'.

\begin{tabular}{|c|c|c|c|c|c|c|c|}
\hline & & & Liking $^{\mathrm{a}}$ & Willingness $^{\mathrm{a}}$ & Ease $^{\mathrm{a}}$ & Difficulties $^{\mathrm{a}}$ & Awareness $^{\mathrm{a}}$ \\
\hline \multirow[t]{4}{*}{2 portions $\mathrm{FV} /$ day $(n=11)$} & Baseline $^{\mathrm{b}}$ & Med & $0 \cdot 2$ & $1 \cdot 0$ & $0 \cdot 8$ & $0 \cdot 2$ & $2 \cdot 0$ \\
\hline & & IQR & $0 \cdot 0,0 \cdot 6$ & $1 \cdot 0,1 \cdot 7$ & $0 \cdot 3,1 \cdot 0$ & $0 \cdot 0,0 \cdot 4$ & $1 \cdot 0,2 \cdot 0$ \\
\hline & Week 4 & Med & $0 \cdot 4$ & $1 \cdot 2$ & $0 \cdot 8$ & $0 \cdot 2$ & $2 \cdot 0$ \\
\hline & & IQR & $0 \cdot 2,0 \cdot 6$ & $1 \cdot 0,1 \cdot 9$ & $0 \cdot 5,0 \cdot 8$ & $0 \cdot 1,0 \cdot 7$ & $1 \cdot 0,2 \cdot 0$ \\
\hline \multirow[t]{4}{*}{5 portions $\mathrm{FV} /$ day $(n=9)$} & Baseline $^{\mathrm{b}}$ & Med & $0 \cdot 2$ & $0 \cdot 8$ & 0.5 & $0 \cdot 4$ & $1 \cdot 0$ \\
\hline & & IQR & $0 \cdot 2,0 \cdot 6$ & $0 \cdot 6,1 \cdot 3$ & $-0 \cdot 1,0 \cdot 6$ & $0 \cdot 0,1 \cdot 3$ & $1 \cdot 0,2 \cdot 0$ \\
\hline & Week 4 & Med & $1 \cdot 0$ & $1 \cdot 5$ & $0 \cdot 5$ & 0.6 & $2 \cdot 0$ \\
\hline & & IQR & $0 \cdot 0,1 \cdot 9$ & $1 \cdot 0,1 \cdot 9$ & $0 \cdot 1,-1 \cdot 0$ & $-1 \cdot 0,1 \cdot 5$ & $1 \cdot 0,2 \cdot 0$ \\
\hline \multirow[t]{4}{*}{8 portions $\mathrm{FV} /$ day $(n=10)$} & Baseline $^{\mathrm{b}}$ & Med & $0 \cdot 3$ & $1 \cdot 00$ & $0 \cdot 8$ & 0.6 & $1 \cdot 5$ \\
\hline & & IQR & $-0 \cdot 4,0 \cdot 9$ & $0 \cdot 5,1 \cdot 5$ & $0 \cdot 4,1 \cdot 3$ & $-0 \cdot 3,0 \cdot 7$ & $1 \cdot 0,2 \cdot 0$ \\
\hline & Week 4 & Med & $1 \cdot 0$ & $1 \cdot 3$ & $0 \cdot 8$ & $0 \cdot 6$ & $2 \cdot 0$ \\
\hline & & IQR & $0 \cdot 0,1 \cdot 5$ & $0 \cdot 9,1 \cdot 7$ & $0 \cdot 3,1 \cdot 0$ & $1 \cdot 0,1 \cdot 0$ & $1 \cdot 0,2 \cdot 0$ \\
\hline$P$ value & & & $0 \cdot 44^{\mathrm{d}}$ & $0 \cdot 46$ & $0 \cdot 62$ & $0 \cdot 44$ & $0 \cdot 25$ \\
\hline
\end{tabular}

${ }^{\text {a }}$ Scores range from -2 (strongly disagree) to 2 (strongly agree). Data are presented as median and interquartile range. ${ }^{b}$ Baseline values did not differ significantly between groups. Significance level was set at $P<0.05$ (Kruskal-Wallis test). ${ }^{\mathrm{c}} P$ value for change in scores (change calculated as week 4 -week 0 scores) by group (Kruskal-Wallis test). ${ }^{\mathrm{d}}$ Significant within-group change from baseline to week 4 (Wilcoxon Signed Rank Test).

This study showed that a four week dietary intervention had no effect on barriers towards FV as assessed by the closed-response questionnaire items, however some changes were detected within the open-ended responses. Larger studies are required to test this hypothesis further.

1. Bates B, Lennox A, Prentice A et al. (2012) National Diet and Nutrition Survey. Headline results from Years 1, 2 and 3 (combined) of the Rolling programme (2008/2009-2010/11). Available at: http://transparency.dh.gov.uk/2012/07/25/ndns-3-years-report/

2. Yeh M-C, Ickes SB, Lowenstein LM et al. (2008) Health PromotInt 23, 42-51.

3. Appleton KM, McGill R, Neville C et al. (2010) Public Health Nutr 13, 514-521. 